\title{
The Magnetized Intracluster Plasma
}

\author{
Jean Eilek \\ New Mexico Tech, Socorro, NM, U.S.A.
}

\begin{abstract}
We now know that the plasma in clusters of galaxies is magnetized, at strong levels in some regions. It follows that the dynamics of the cluster plasma is more complex than has been thought up to now.
\end{abstract}

\section{Introduction}

Clusters of galaxies contain a plasma atmosphere - the intracluster medium (ICM). The hot, thermal component of the ICM is detected in X-rays. Early studies envisioned the ICM as sitting quietly in a static cluster potential. However, our picture is changing as evidence shows that clusters, and the ICM therein, are still evolving at the present epoch. We now know also that the ICM contains relativistic particles and magnetic fields, intermixed with the thermal component. In this paper I concentrate on the magnetic field, but emphasize that it should be seen as one component of the dynamic ICM.

\section{Observations of the Magnetized ICM}

Diffuse synchrotron radio haloes have been found in several clusters (Feretti \& Giovannini 1996), and it appears likely that ongoing work (such as Giovannini et al. 1999) will find quite a few more. The detection of a cluster-wide radio halo is, of course, a detection of a cluster-wide magnetic field. The field strength cannot be found directly from such an observation. One must make some assumption, such as equipartition, to separate the magnetic and relativistic particle densities. Existing work finds equipartition fields $\sim 0.7 \mu \mathrm{G}$ (assuming homogeneous magnetic fields, which is probably too simple a picture).

Faraday rotation measure (RM) has now been determined for a large number of radio sources within and behind clusters. Clarke et al. (2000) is the latest statistical work. They find excess RM for sources in and behind clusters, and infer a typical cluster field $\sim 5 \mu$ G. Eilek \& Owen (2000; "EO") present resolved RM images of cluster-center sources. They combine their data with previous work to find typical scale lengths $\sim 10 \mathrm{kpc}$, and fields on the order of tens of $\mu \mathrm{G}$ in cluster cores.

Taken together, these results suggest that cluster-wide fields are common, at a level of a few $\mu \mathrm{G}$. This gives a pressure ratio, $\beta=B^{2} / 8 \pi n k_{B} T \sim 0.1$, for a "typical cluster". In addition, dynamically significant fields exist in cluster cores. EO present $\beta$ values for each of their 10 clusters; the typical range is $\beta \sim 1$. What can be said about the field structure? The RM images show 
(resolved) order scales up to $\sim 10 \mathrm{kpc}$, measured out to $\sim 100 \mathrm{kpc}$ in the cluster core. Cluster-wide radio haloes are strongly depolarized, which suggests that the field is disordered on scales small compared to the observing beam or the path through the cluster. Study of other magnetized plasmas (e.g., Eilek 1999) suggests that the cluster field is very likely to be filamented, with high-field flux ropes separated by lower-field regions.

\section{Physics of the Magnetized ICM}

Two physical questions arise: what supports the magnetic field, and what consequences does the field have for the ICM?

\subsection{Drivers}

It is most likely that the magnetic field is supported by some sort of dynamo - that is, bulk flows or turbulence in the plasma. This hypothesis seems most consistent with our growing sense that the ICM is dynamic and turbulent. (In this section I summarize arguments from EO.) Studies of turbulent dynamos show that a field $B^{2} \sim 4 \pi \rho v^{2}$ can be reached after several eddy times, which is fast enough to be relevant if the turbulence is transonic and moderate-scale.

What drives the turbulence? We can identify three possibilities. (1) Driving by embedded active galaxies can support $\beta \sim 1$ fields in a region local to the galaxy. (2) Cooling-driven collapse will enhance weak seed fields and can reach $\beta \sim 1$ levels. (3) Ongoing mergers, even minor ones, can be a very strong driver, and should be able to maintain strong fields on a cluster-wide scale. (Galaxy driving is not strong enough to maintain the observed field levels.) The first two processes are local ones; but it could be argued that cluster-wide magnetic fields are an indirect detection of ongoing evolution of the cluster potential.

\subsection{Consequences}

What role does the magnetic field play in the dynamics of the ICM? There are at least four areas worthy of further study.

Microscopic transport. Large-scale dynamics of the ICM depend on smallscale transport processes, which can be dramatically affected even by a low- $\beta$ magnetic field. Simple cooling-flow models require strong suppression of thermal conductivity. Some authors believe this can be achieved through small-scale field-line tangling, while others do not. Some radio halo models require the relativistic electrons to propagate from a localized source throughout the cluster. Disordered magnetic fields can effectively trap the particles, thus distributed sources may be required. These arguments continue at the present time. Their resolution appears to require better knowledge of the small-scale field structure (say the correlation length) than we have as yet.

Energy flow. The thermodynamics of the ICM will also be changed by the magnetic field. If the ICM were an unmagnetized, single-phase plasma, then energy flow would be simple. Energy comes in from gravitational collapse (both early and ongoing), with possible additions from active galaxies; and leaves as radiation. Energy is stored in the plasma as internal energy. If the ICM is 
magnetized, the story becomes much richer. Energy can also be stored inductively, in the magnetic field, which can be enhanced through bulk flows and gravitational collapse. This stored energy can be released through reconnection events, and can go to heating of the ICM, or to relativistic particle acceleration. In addition, magnetized plasma-wave turbulence can couple large-scale kinetic energy to relativistic particles, through turbulent acceleration of those particles.

Hydrostatic support. A strong $(\beta \sim 1)$ magnetic field will contribute to the pressure support of the ICM. We now know this must be part of the picture in cluster cores, including several strong cooling cores (as in EO). This fact has immediate impact in two areas. (1) The situation in strong cooling cores must be revisited. The fields in some of these cores are strong enough to support the gas in the gravitational field of the cluster. It may be that the gas in these cores is cooling but not collapsing (cf. Garasi \& Eilek 2001; Markovic \& Eilek 2001). (2) The pressure profile of the thermal plasma does not provide an accurate measure of the gravitational potential of the cluster. Dark matter determinations which rely only on the thermal gas will be underestimated, at least in the cluster cores.

Instabilities and turbulence. A hydrostatic situation in which the gas is even partially supported against gravity by a magnetic field is very unlikely to be stable. Magnetic buoyancy and Parker-type instabilities must come into play. Such instabilities will keep the plasma "stirred up" and turbulent. One would expect these effects to be particularly important in the $\beta \sim 1$ environment of cluster cores. (If the core also contains an active radio galaxy, which deposits energy into the local ICM, the magnetic field will mediate the effects of that energy deposition.) We may be seeing examples of this process in new X-ray images of cooling cores such as Perseus or A2199.

Acknowledgments. My understanding of the magnetized ICM has benefited greatly from ongoing discussions with several colleagues, including $\mathrm{T}$. Clarke, D. De Young, C. Garasi, T. Markovic, F. Owen and J. Weatherall. This work has been partially supported by NSF grant AST9720263.

\section{References}

Clarke, T., Kronberg, P.P. \& Böhringer, H. 2000, submitted to ApJ

Eilek, J.A. 1999, in Diffuse Thermal and Relativistic Plasma in Galaxy Clusters, eds. H. Böhringer, L. Feretti, \& P. Scheucker (MPE Report 271), 71

Eilek, J.A. \& Owen, F.N. 2000, submitted to ApJ (EO)

Feretti, L. \& Giovannini, G. 1996, in Extragalactic Radio Sources, eds. R. Ekers, C. Fanti \& L. Padrielli, 333

Garasi, G.J., \& Eilek, J.A. 2001, in preparation

Giovannini, G., Tordi, M. \& Feretti, L. 1999, New Astronomy, 4, 141

Markovic, T. \& Eilek, J A. 2001, in preparation 